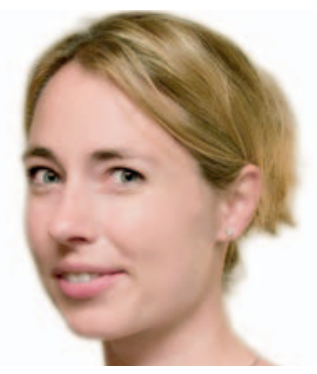

\title{
Metastasiertes Magenkarzinom - längeres Überleben durch Resektion?
}

\author{
Sylvie Lorenzen
}

III. Medizinische Klinik, Klinikum rechts der Isar, Technische Universität München, München, Deutschland

\begin{abstract}
Al-Batran SE, Homann N, Pauligk C, Illerhaus G, Martens UM, Stoehlmacher J, Schmalenberg H, Luley KB, Prasnikar N, Egger M, Probst S, Messmann H, Moehler M, Fischbach W, Hartmann JT, Mayer F, Höffkes HG, Koenigsmann M, Arnold D, Kraus TW, Grimm K, Berkhoff S, Post S, Jäger E, Bechstein W, Ronellenfitsch U, Mönig S, Hofheinz RD: Effect of neoadjuvant chemotherapy followed by surgical resection on survival in patients with limited metastatic gastric or gastroesophageal junction cancer: the AIO-FLOT3 trial. JAMA Oncol 2017;3:1237-1244.
\end{abstract}

\section{Summary}

Importance: Surgical resection has a potential benefit for patients with metastatic adenocarcinoma of the stomach and gastroesophageal junction.

Objective: To evaluate outcome in patients with limited metastatic disease who receive chemotherapy first and proceed to surgical resection.

Design, Setting, and Participants: The AIO-FLOT3 (Arbeitsgemeinschaft Internistische Onkologie-fluorouracil, leucovorin, oxaliplatin, and docetaxel) trial is a prospective, phase 2 trial of 252 patients with resectable or metastatic gastric or gastroesophageal junction adenocarcinoma. Patients were enrolled from 52 cancer care centers in Germany between February 1, 2009, and January 31, 2010, and stratified to 1 of 3 groups: resectable (arm A), limited metastatic (arm B), or extensive metastatic (arm C). Data cutoff was January 2012, and the analysis was performed in March 2013.

Interventions: Patients in arm A received 4 preoperative cycles of fluorouracil, leucovorin, oxaliplatin, and docetaxel (FLOT) followed by surgery and 4 postoperative cycles. Patients in arm B received at least 4 cycles of neoadjuvant FLOT and proceeded to surgical resection if restaging (using computed tomography and magnetic resonance imaging) showed a chance of margin-free (RO) resection of the primary tumor and at least a macroscopic complete resection of the metastatic lesions. Patients in arm C were offered FLOT chemotherapy and surgery only if required for palliation. Patients received a median (range) of 8 (1-15) cycles of FLOT.

Main Outcomes and Measures: The primary end point was overall survival.

Results: In total, 238 of 252 patients (94.4\%) were eligible to participate. The median (range) age of participants was 66 (36-79) years in arm A ( $n=51), 63$ (28-79) years in arm B ( $n=60)$, and 65 (23-83) years in arm $C(n=127)$. Patients in arm $B(n=60)$ had only retroperitoneal lymph node involvement (27 patients, 45\%), liver involvement (11, 18.3\%), lung involvement (10, 16.7\%), localized peritoneal involvement $(4,6.7 \%)$, or other $(8,13.3 \%)$ incurable sites. Median overall survival was 22.9 months $(95 \% \mathrm{Cl}, 16.5$ to upper level not achieved) for arm B, compared with 10.7 months ( $95 \% \mathrm{Cl}$, 9.1-12.8) for arm C (hazard ratio, 0.37; 95\% Cl, 0.25-0.55) ( $<<0.001)$. The response rate for arm B was 60\% (complete, 10\%; partial, 50\%), which is higher than the $43.3 \%$ for arm C. In arm B, 36 of 60 patients (60\%) proceeded to surgery. The median overall survival was 31.3 months (95\% Cl, 18.9-upper level not achieved) for patients who proceeded to surgery and 15.9 months ( $95 \%$ Cl, 7.1-22.9) for the other patients.

Conclusions and Relevance: Patients with limited metastatic disease who received neoadjuvant chemotherapy and proceeded to surgery showed a favorable survival. The AIO-FLOT3 trial provides a rationale for further randomized clinical trials.

Trial Registration: clinicaltrials.gov identifier: NCT00849615.

๑) 2017 American Medical Association, Chicago

\section{KARGER}

(C) 2018 S. Karger GmbH, Freiburg 


\section{Transfer in die Praxis}

\section{Hintergrund}

Die systemische Chemotherapie verbessert sowohl das Überleben als auch die Lebensqualität von Patienten mit fortgeschrittenem Magenkarzinom gegenüber einer besten supportiven Behandlung. Dennoch liegt die mediane Überlebenszeit für diese Patienten in der westlichen Hemisphäre bislang unter 1 Jahr [1] und das 2-Jahresüberleben unter 10\%. Im Gegensatz zu anderen Tumoren wie z.B. dem Kolon- oder Ovarialkarzinom, die im Falle einer limitierten Metastasierung multimodalen Behandlungskonzepten zugeführt werden, ist das Behandlungsziel beim metastasiertem Magenkarzinom, unabhängig vom Ausmaß der Metastasierung, rein palliativ. Derzeit wird eine Resektion von Primärtumor und Metastasen außerhalb von Studien nicht empfohlen, obwohl sich die Hinweise in Publikationen häufen, dass einige, selektionierte Patienten mit metastasiertem Magenkarzinom unter bestimmten Voraussetzungen offensichtlich von einer Resektion des Primärtumors und der Metastasen profitieren. Dies betrifft insbesondere Patienten mit limitierter Metastasierung - definiert als solitäre resektable Organmetastasen, die komplett (RO) reseziert werden können -, mit gutem Allgemeinzustand, keinem oder nur limitierten Nachweis einer Peritonealkarzinose und einem guten Ansprechen der Tumormanifestationen auf eine vorangegangene systemische Chemotherapie. Problematisch bleiben allerdings die geringen Fallzahlen, die sehr heterogenen Patientenkollektive z.T. ohne exakte Definition der Metastasierungsmuster und die unterschiedlichen Therapiekonzepte in den einzelnen Studien und retrospektiven Analysen. Daten für den Erfolg einer Induktionschemotherapie mit anschließender Resektion von Primarius und Metastasen gibt es kaum. Die kürzlich veröffentlichte asiatische REGATTA-Studie konnte keinen Überlebensvorteil für die Resektion des Primärtumors bei limitiert metastasiertem Magenkarzinom gegenüber einer alleinigen palliativen Chemotherapie zeigen [2], wobei in dieser Studie die Metastasen nicht mitreseziert wurden, sodass durch das Studiendesign keine Aussagen zur Sinnhaftigkeit der synchronen Metastasenresektion gemacht werden kann.

\section{Studienergebnisse}

Auf der Basis einer großen prospektiv geführten Kohorte im Rahmen der Phase-II-FLOT3-Studie konnten jetzt Überlebensdaten von Patienten mit synchron limitiert metastasiertem Adenokarzinom des Magens oder des gastroösophagealen Übergangs und Resektion nach präoperativer Induktionstherapie mit FLOT erhoben werden.

Die zwischen Februar 2009 und Januar 2010 durchgeführte FLOT3Studie umfasste 252 konsekutive Patienten, von denen 238 auswertbar waren: 51 Patienten mit lokal fortgeschrittener Erkrankung erhielten eine neoadjuvante Chemotherapie gefolgt von Resektion (Kohorte A), 60 Patienten mit limitierter Metastasierung konnten nach einer Induktionstherapie sekundär reseziert werden (Kohorte B) und 127 Patienten wurden bei ausgedehnter Metastasierung rein palliativ chemotherapiert (Kohorte C). Alle Patienten erhielten, unabhängig von der vom Studienleiter zugeteilten Kohorte, eine Chemotherapie nach dem FLOT-Regime. Für die Patienten in Kohorte B war die Definition für limitierte Metastasierung wie folgt festgelegt: ausschließlich lymphogene Metastasierung oder/und eine potentiell resektable/lokal behandelbare Organmetastasierung mit oder ohne lymphogene retroperitoneale Metastasierung. Die Mehrzahl der Patienten in Kohorte B wiesen eine ausschließlich retroperitoneale, lymphogene Metastasierung (45\%), gefolgt von Lebermetastasierung (18.3\%) auf.

Patienten in Kohorte B mit limitierter Metastasierung konnten, nach einer Induktionstherapie mit 4 Zyklen FLOT und wenn die Erkrankung keinen Progress zeigte, einer sekundären Resektion von Primärtumor und Metastasen zugeführt werden. Insgesamt wurde eine Resektion bei $60 \%$ der Patienten durchgeführt.

Nach einer medianen Nachbeobachtungszeit von 28,6 Monaten zeigte sich eine Verdopplung des medianen Überlebens in der Kohorte B im Vergleich zu den nicht resezierten Patienten mit ausschließlich systemischer Therapie (Kohorte C) (22,9 vs. 10,7 Monate). Innerhalb der Kohorte B lag das mediane Überleben der resezierten Patienten bei 31,3 Monaten im Vergleich zu 15,9 Monaten bei den Patienten, die keine Resektion erhalten hatten. Ebenso war das progressionsfreie Überleben bei den resezierten Patienten im Vergleich zu den nicht-resezierten Patienten deutlich verlängert (26,7 Monate vs. 8,4 Monate). Innerhalb der Subgruppen mit limitierter Metastasierung hatte die Gruppe mit ausschließlich retroperitonealer Metastasierung den größten Vorteil von der Resektion im Vergleich zu der Subgruppe der hepatisch metastasierten Patienten, die den geringsten Vorteil aufwies. Insgesamt war das Überleben deutlich länger als was aus Studien mit rein palliativ intendierter Chemotherapie zu erwarten ist (9-11 Monate). Allerdings stellt sich die Frage, welchen Anteil die Resektion an dem günstigeren Überleben hat. Da es sich nicht um eine randomisierte Studie handelt, ist von einem hohen Selektionsbias auszugehen.

Trotz der beachtlichen Überlebenszeit von 31 Monaten bei resezierten Patienten ist diese Studie ausschließlich Hypothesen generierend, da in der Regel nur Patienten mit gutem PerformanceStatus und günstiger Prognose reseziert werden. Eine endgültige Beurteilung der Frage lässt sich daher mit der derzeitig verfügbaren Datenlage nicht treffen.

\section{Fazit für die Praxis}

Die prospektive Studie von Al-Batran et al. ermöglicht den Schluss, dass eine Resektion von Metastasen und Primärtumor nach einer vorangegangenen Chemotherapie sinnvoll sein kann und bei selektionierten Patienten mit limitierter metastasierter Erkrankung zu einer signifikanten Verlängerung des Überlebens führt. Obwohl die publizierten Daten z.T. erfolgsversprechend zu sein scheinen, müssen sie zum jetzigen Zeitpunkt mit Vorsicht interpretiert werden und müssen erst durch randomisierte Studien bestätigt werden. Diese Analyse unterstreicht die Notwendigkeit für zusätzliche prospektive Evidenz, die uns die Entscheidung pro/contra Resektion 
eines Magenkarzinoms mit resektablen Metastasen erleichtert. Die randomisierte Phase-III-RENAISSANCE-Studie [3] evaluiert bei Patienten, die nach einer Induktionstherapie mit 4 Zyklen FLOT keinen Progress zeigen, den Stellenwert der Resektion gegenüber einer Fortführung der systemischen Chemotherapie. Primärer Endpunkt ist das Gesamtüberleben. Insgesamt sollen 271 Patienten eingeschlossen werden, von denen mindestens 176 Patienten in einen der Behandlungsarme randomisiert werden sollen.

\section{Disclosure Statement}

Hiermit erkläre ich, dass keine Interessenskonflikte in Bezug auf den vorliegenden Kommentar bestehen.

\section{Literatur}

1 Lordick F, Lorenzen S, Yamada Y, Ilson D: Optimal chemotherapy for advanced gastric cancer: is there a global consensus? Gastric Cancer 2014;17: 213-225.

2 Fujitani K, Yang HK, Mizusawa J, et al.: REGATTA study investigators. Gastrectomy plus chemotherapy versus chemotherapy alone for advanced gastric cancer with a single non-curable factor (REGATTA): a phase 3, randomised controlled trial. Lancet Oncol 2016;17:309-318.

3 Al-Batran SE, et al.: Chemotherapy alone vs. chemotherapy + surgical resection in patients with limited-metastatic adenocarcinoma of the stomach or esophagogastric junction (FLOT5) (RENNAISSANCE). NCT02578368.

Kontaktadresse: PD Dr. Sylvie Lorenzen, III: Medizinische Klinik, Klinikum rechts der Isar, Technische Universität München, Ismaninger Straße 22, 81675 München, Deutschland,

sylvie.lorenzen@mri.tum.de 